\title{
THE DERIVATIVE OF A BOUNDED HOLOMORPHIC FUNCTION IN THE DISK
}

\author{
SHIN J YAMASHITA
}

ABSTRACT. Let a nonconstant function $f$ be holomorphic and bounded, $|f|<1$ in $D:|z|<1$. We shall estimate $f^{*}(z)=\left(1-|z|^{2}\right)\left|f^{\prime}(z)\right| /\left(1-|f(z)|^{2}\right)$ at each point $z \in D((1)$ in Theorem 1$)$. The function $d$ appearing in the estimate concerns the sizes of the schlicht disks on the Riemannian image $\mathcal{F}$ of $D$ by $f$. Boundary properties of $f$ and $f^{*}$ will be stated in Theorems 2 and 3 ; use is made of the cluster sets of $d$.

1. Results. Consider the metric

$$
\delta(w, z)=|w-z| /|1-\bar{z} w| \quad(z, w \in D)
$$

in $D(\delta(w, z)=[w, z]$ in $[8$, p. $510 \mathrm{ff}$.]). Denote $D(z, t)=\{w \in D ; \delta(w, z)<t\}$ $(z \in D, 0<t \leq 1)$. By $f$ we always mean a nonconstant function holomorphic and bounded, $|f|<1$ in $D$. Let $d(z) \equiv d(z, f)$ be the maximum of $t$ such that $\mathcal{F}$, covering $D$, contains the schlicht $D(f(z), t)$ of center $f(z) \in \mathcal{F}$; if such a $t$ does not exist we set $d(z)=d(z, f)=0$. We then have $0 \leq d(z) \leq 1$ at each $z \in D$ and further, $d(z)=1$ at (each) $z \in D$ if and only if $f$ is schlicht from $D$ onto $D$. Plainly, $d(z)=0$ if and only if $f^{\prime}(z)=0$.

Theorem 1. At each $z \in D$ we have

$$
d(z, f) \leq f^{*}(z) \leq\{8 d(z, f)\}^{1 / 2} .
$$

Since $f^{*}(z) \leq 1$ at each $z \in D$ by the lemma of $H$. A. Schwarz and G. Pick, the right-hand side of (1) has the meaning if $d(z, f)<1 / 8$. Theorem 1 is analogous to the result of $\mathrm{Ch}$. Pommerenke [7, Theorem 1] who uses the Euclidean distance to measure the sizes of the schlicht disks on $\mathcal{F}$.

By an angular domain at a point $\zeta$ of $\Gamma:|z|=1$, we mean a triangular domain whose vertices are $\zeta$ and two points of $D$. Dy an admissible arc at $\zeta$ we mean a continuous curve $\Lambda: z=z(t) \in D(0 \leq t<1), \lim _{t \rightarrow 1} z(t)=\zeta$, tangent at $\zeta$ to a chord of $\Gamma$ at $\zeta$. We call $\zeta \in \Gamma$ of first kind if

$$
\lim _{z \rightarrow \zeta, z \in \mathbf{\Lambda}} f^{*}(z)=0
$$

for each admissible arc $\Lambda$ at $\zeta$, while we call $\zeta \in \Gamma$ of second kind if $\lim \inf _{z \rightarrow \zeta, z \in \Delta} f^{*}(z)>0$ for each angular domain $\Delta$ at $\zeta$. A point $\zeta \in \Gamma$ is

Received by the editors May 14, 1974 and, in revised form, September 11, 1974 . AMS (MOS) subject classifications (1970). Primary 30A 72.

Key words and phrases. Riemannian image, schlicht disks, non-Euclidean distance, the lemma of Schwarz and Pick, cluster sets. 
called of third kind if $\lim \inf _{z \rightarrow \zeta, z \in D} f^{*}(z)>0$. Let $S_{1}(f), S_{2}(f)$ and $S_{3}(f)$ be the sets of all points of first, second, and third kind, respectively. Plainly, $S_{3}(f) \subset S_{2}(f)$ and $S_{1}(f) \cap S_{2}(f)=\varnothing$ (empty). Our next results are two.

Theorem 2. The union $S_{1}(f) \cup S_{2}(f)$ is of linear measure $2 \pi$ and residual $[2, p .75]$ on $\Gamma$, and the union $S_{1}(f) \cup S_{3}(f)$ is residual on $\Gamma$.

Let

$$
\sigma(w, z)=1 / 2 \log \{(1+\delta(w, z)) /(1-\delta(w, z))\}
$$

be the non-Euclidean distance of $w$ and $z$ of $D$. Let $l(w, z ; f)(w, z \in D)$ be the non-Euclidean length of the Riemannian image by $f$ of the (Euclidean) line segment joining $w$ and $z$.

Theorem 3. If $\Delta$ is an angular domain at $\zeta \in S_{2}(f)$, then there exist an open disk $U$ containing $\zeta$ and a constant $k_{1}>0$ such that $k_{1} \sigma(w, z) \leq$ $l(w, z ; f)$ for each pair of points $w, z \in \Delta \cap U$. If $\zeta \in S_{3}(f)$, then there exist an open disk $V$ containing $\zeta$ and a constant $k_{2}>0$ such that $k_{2} \sigma(w, z) \leq$ $l(w, z ; f)$ for each pair of points $w, z \in D \cap V$.

2. Proof of Theorem 1. We have only to prove (1) for $z$ with $d(z)>0$. The function

$$
g(w)=\frac{f((w+z) /(1+\bar{z} w))-f(z)}{1-\overline{f(z)} f((w+z) /(1+\bar{z} w))}
$$

is holomorphic and bounded, $|g|<1$ in $|w|<1$ with $g(0)=0$ and $\left|g^{\prime}(0)\right|=$ $f^{*}(z) \neq 0$. Therefore the function $h(w) \equiv g(w) / g^{\prime}(0)=w+a_{2} w^{2}+\cdots$ is bounded by $M=1 /\left|g^{\prime}(0)\right|$ in $|w|<1$. To the function $h$ we may apply the result [8, Corollary, p. 261] deduced from the theorem [8, Theorem VI.10, p. 259] due to J. Dieudonné [3, p. $349 \mathrm{ff}$.]. Then the Riemannian image of $|w|<1$ by $h$ contains the schlicht disk $D(0,1 / 8 M)=D\left(0,\left|g^{\prime}(0)\right| / 8\right)$, whence the Riemannian image of $|w|<1$ by $g$ contains the schlicht disk $D\left(0,\left|g^{\prime}(0)\right|^{2} / 8\right)$. Therefore $\mathcal{F}$ contains the schlicht disk $D\left(f(z),\left|g^{\prime}(0)\right|^{2} / 8\right)$, whence $f^{*}(z)^{2} / 8=\left|g^{\prime}(0)\right|^{2} / 8$ $\leq d(z)$. This proves the right-hand side of (1). Let $F$ be the inverse of $f$ in $D(f(z), d(z))$ such that $F(f(z))=z$. The function

$$
G(w)=\frac{F((d(z) w+f(z)) /(1+\overline{f(z)} d(z) w))-z}{1-\bar{z} F((d(z) w+f(z)) /(1+\overline{f(z)} d(z) w))}
$$

is holomorphic and bounded, $|G|<1$ in $|w|<1$ with $G(0)=0$. Consequently, by Schwarz' lemma, $d(z) / f^{*}(z)=\left|G^{\prime}(0)\right| \leq 1$, from which follows the left-hand side of (1).

3. Proofs of Theorems 2 and 3. We begin with

Lemma 1. For each pair $w, z \in D$, 


$$
|d(w)-d(z)| \leq \sigma(w, z) .
$$

Proof. (The present proof, due to the referee, is more concise than the original one.) Without loss of generality we may assume that $d(z)<d(w)$. Then, $d(w)<1$, for otherwise, $d(z)=d(w)=1$. We may further assume $\sigma(w, z)$ $<d(w)$, for otherwise, (2) is trivial. Since $\delta(f(w), f(z)) \leq \delta(w, z) \leq \sigma(w, z)$ $<d(w), f(z)$ lies in the disk $D(f(w), d(w))$. Hence the two disks $D(f(w), d(w))$ and $D(f(z), d(z))$ must possess a common boundary point $v \in D$, so that $\delta(f(w), v)=d(w)$ and $\delta(f(z), v)=d(z)$. It follows from $d(z)<d(w)$ that $\sigma(f(z), v)<\sigma(f(w), v)$. Now, making use of the fact that $\delta(f(w), v)=$ $\tanh \sigma(f(w), v), \delta(f(z), v)=\tanh \sigma(f(z), v)$, the fact that $\tanh A-\tanh B \leq$ $A-B$ for $A \geq B \geq 0$, the triangle inequality for the metric $\sigma$, and the SchwarzPick lemma, we obtain the following chain of inequalities:

$$
\begin{aligned}
d(w)-d(z) & =\delta(f(w), v)-\delta(f(z), v) \leq \sigma(f(w), v)-\sigma(f(z), v) \\
& \leq \sigma(f(w), f(z)) \leq \sigma(w, z) .
\end{aligned}
$$

This completes the proof of the lemma.

Let $F$ be a subset of $D$ whose closure $\bar{E}$ in the plane contains a point $\zeta \in \Gamma$. Then we set $C_{E}(d, \zeta)=\bigcap_{U} \overline{d(E \cap U)}$, where $U$ ranges over all open disks containing $\zeta$. The cluster set $C_{E}(d, \zeta)$ relative to $E$ lies in the closed interval $[0,1]$. We set $C^{A}(d, \zeta)=\bigcup_{\Delta} C_{\Delta}(d, \zeta)$ and $\Pi_{T}(d, \zeta)=$ $\bigcap_{\boldsymbol{\Lambda}} C_{\boldsymbol{\Lambda}}(d, \zeta), \Delta$ ranging over all angular domains at $\zeta$ and $\Lambda$ ranging over all admissible arcs at $\zeta$. Let $K(d)$ be the set of all points $\zeta \in \Gamma$ such that $C^{A}(d, \zeta)=C_{\Delta}(d, \zeta)$ for each angular domain $\Delta$ at $\zeta$ and let $J(d)$ be the set of all points $\zeta \in K(d)$ satisfying $C^{A}(d, \zeta)=C_{D}(d, \zeta)$. Finally, let $L(d)$ be the set of all points $\zeta \in \Gamma$ such that $C^{A}(d, \zeta)=\Pi_{T}(d, \zeta)$.

Lemma 2. $J(d) \subset K(d)=L(d)$

Proof. $J(d) \subset K(d)$ follows from the definition of $J(d)$. Since each angular domain at $\zeta \in L(d)$ contains a terminal part of a chord of $\Gamma$ at $\zeta$, it follows from $C^{A}(d, \zeta)=\Pi_{T}(d, \zeta)$ that $C^{A}(d, \zeta)=C_{\Delta}(d, \zeta)$ for each a ngular domain $\Delta$ at $\zeta$, that is, $\zeta \in K(d)$. Thus $L(d) \subset K(d)$. To prove $L(d) \supset K(d)$, we remark that $d$ is uniformly continuous as a map from $D$ endowed with the metric $\sigma(\cdot, \cdot)$ into $[0,1]$. The proof is therefore the same as that of $[1$, Lemma].

Proof of Theorem 2. First of all, we may replace $f^{*}(z)$ by $d(z, f)$ in the definition of $S_{j}(f), j=1,2,3$; this is a consequence of (1). We shall prove

$$
\begin{aligned}
& K(d) \subset S_{1}(f) \cup S_{2}(f) ; \\
& J(d) \subset S_{1}(f) \cup S_{3}(f) .
\end{aligned}
$$


By Lemma 2, $K(d)=L(d)$. If $\left.\zeta \in K^{(} d\right)$ and $0 \in C^{A}(d, \zeta)$, then $0 \in \Pi_{T}(d, \zeta)$, whence $\zeta \in S_{1}(f)$, while if $\zeta \in K(d)$ and $0 \notin C^{A}(d, \zeta)$, then $0 \notin C_{\Delta}(d, \zeta)$ for each $\Delta$ at $\zeta$, whence $\zeta \in S_{2}(f)$. We thus obtain (3). If $\zeta \in J(d)$ and $0 \epsilon$ $C_{D}(d, \zeta)$, then $0 \in \Pi_{T}(d, \zeta)=C^{A}(d, \zeta)=C_{D}(d, \zeta)$ because $J(d) \subset L(d)$. Therefore $\zeta \in S_{1}(f)$. If $\zeta \in J(d)$ and $0 \notin C_{D}(d, \zeta)$, then $\zeta \in S_{3}(f)$. We thus have (4). Now, according to the results on arbitrary functions (cf. [6, Theorems 1 and 2]), $K(d)$ is of measure $2 \pi$ and $J(d)$ is residual on $\Gamma$. Hence $K(d) \supset J(d)$ is residual. Theorem 2 now follows from (3) and (4).

Remark. Let a real function $q$ in $D$ be uniformly continuous with respect to $\sigma$. Then $J(q) \subset K(q)=L(q)$ by the identical reasoning as in the proof of Lemma 2. Assume further that $q(z) \geq 0$ for all $z \in D$. If $q$ replaces $f^{*}$ in the definitions of $S_{j}(f)(j=1,2,3)$, and if the resulting sets are denoted by $S_{j}(q)(j=1,2,3)$, then Theorem 2 for the present $q$ remains valid by the same proof. Theorem 1 reveals the link between $f^{*}$ and $d$, the special case of $q$.

Proof of Theorem 3. Let $U$ be an open disk containing $\zeta$ such that $k_{\mathrm{I}}=$ inf $z \in \Delta \cap U) f^{*}(z)>0$. It then follows that

$$
k_{1}|d \xi| /\left(1-|\xi|^{2}\right) \leq\left|f^{\prime}(\xi)\right||d \xi| /\left(1-|f(\xi)|^{2}\right)
$$

for each $\xi \in \Delta \cap U$. Since $k_{1} \sigma(w, z)$ is obtained by integrating the left-hand side of (5) along the geodesic line connecting $w$ and $z, k_{1} \sigma(w, z)$ is less than the integral of the left-hand side along the Euclidean line segment joining $z$ and $w$, being contained in the convex set $\Delta \cap U$. We thus obtain $k_{1} \sigma(w, z) \leq l(w, z ; f)$. The proof of the rest is similar.

4. A special class of functions. We consider the distribution of $S_{1}(f)$ of a special $f$. Suppose

$$
\iint_{|z|<1}\left(\frac{\left|f^{\prime}(z)\right|}{1-|f(z)|^{2}}\right)^{p} d x d y<+\infty \quad(1 \leq p<+\infty, z=x+i y) .
$$

Consider the case $p=1$. By G. Fubini's theorem with $1 / 2 \leq r$, we obtain

$$
\int_{1 / 2}^{1} \frac{\left|f^{\prime}(\tau \zeta)\right|}{1-|f(\tau \zeta)|^{2}} d r<+\infty
$$

for a.e. (almost every) $\zeta$ on $\Gamma$. Now (7) means that the non-Euclidean length of the Riemannian image of half the radius at $\zeta$ by $f$ is finite. Hence, $f$ has the radial limit $f(\zeta) \in D$ at $\zeta$, which is the angular limit by the theorem of E. Lindelöf [2, Theorem 2.3, p. 19]. By a geometrical consideration on $d$ combined with (1) we have

$$
\lim _{z \rightarrow \zeta, z \in \Delta} d(z, f)=\lim _{z \rightarrow \zeta, z \in \Delta} f^{*}(z)=0
$$

for each angular domain $\Delta$ at $\zeta$; in effect, $d(z, f) \leq \delta(f(z), f(\zeta))$ for $z \in \Delta$ 
near $\zeta$. Thus, $S_{1}(f)$ is of measure $2 \pi$. The case $p>1$. The function

$$
\left|f^{\prime}(z)\right| /\left(1-|f(z)|^{2}\right)=\exp \circ \log \left\{\left|f^{\prime}(z)\right| /\left(1-|f(z)|^{2}\right)\right\}
$$

is nonnegative subharmonic in $D$; actually, the exponential function is convex and even $\log \left\{\left|f^{\prime}(z)\right| /\left(1-|f(z)|^{2}\right)\right\}$ is subharmonic because

$$
\left(\frac{\partial^{2}}{\partial x^{2}}+\frac{\partial^{2}}{\partial y^{2}}\right) \log \left(\frac{\left|f^{\prime}(z)\right|}{1-|f(z)|^{2}}\right)=4\left(\frac{\left|f^{\prime}(z)\right|}{1-|f(z)|^{2}}\right)^{2}>0
$$

at each $z=x+i y$ with $f^{\prime}(z) \neq 0$ (cf. [5, p. 83]). By the result of $\mathrm{F}$. W. Gehring [4, Theorem 1], for a.e. $\zeta \in \Gamma$ we have

$$
\lim _{z \rightarrow \zeta, z \in \Delta} \frac{(1-|z|)^{1 / p}\left|f^{\prime}(z)\right|}{1-|f(z)|^{2}}=0
$$

for each angular domain $\Delta$ at $\zeta$. Consequently,

$$
\lim _{z \rightarrow \zeta, z \in \Delta}(1-|z|)^{(1 / p)-1} f^{*}(z)=0
$$

for each angular domain $\Delta$ at $\zeta$. Hence $f^{*}$ tends to zero rapidly as (9) shows in this case. It should be noted that for $p=2$ (hence for $p \geq 2$ ), we have (7) and, hence, (8) for each $\zeta \in \Gamma$ except for a set of capacity zero on $\Gamma$ (cf. $[9$, Theorem for $j=3]$ ). Therefore $\Gamma-S_{1}(f)$ is of capacity zero.

\section{REFERENCES}

1. Frederick Bagemihl, Some approximation theorems for normal functions, Ann. Acad. Sci. Fenn. Ser. A I No. 335 (1963), 5 pp. MR $28 \# 2219$.

2. Edward F. Collingwood and Arthur J. Lohwater, The theory of cluster sets, Cambridge Tracts in Math. and Math. Phys., no. 56, Cambridge Univ. Press, Cambridge, 1966. MR 38 \#325.

3. Jean Dieudonné, Recherches sur quelques problèmes relatifs aux polynomes et aux fonctions bornées d'une variable complexe, Ann. Sci. École Norm. Sup. 48 (1931), 247-358.

4. Frederick W. Gehring, On the radial order of subharmonic functions, J. Math. Soc. Japan 9 (1957), 77-79. MR 19, 131.

5. Maurice Heins, Selected topics in the classical theory of functions of a complex variable, Athena Series: Selected Topics in Math., Holt, Rinehart and Winston, New York, 1962. MR 29 \#217.

6. Peter A. Lappan, A property of angular cluster sets, Proc. Amer. Math. Soc. 19 (1968), 1060-1062. MR 37 \#6462.

7. Christian Pommerenke, On Bloch functions, J. London Math. Soc. (2) 2 (1970), 689-695. MR 44\#1799.

8. Masatsugu Tsuji, Potential theory in modern function theory, Maruzen, Tokyo, 1959. MR $22 \# 5712$.

9. Shinji Yamashita, Function-theoretic metrics and boundary behaviour of functions meromorphic or holomorphic in the wnit disk, Nagoya Math. J. 45 (1972), 109117. MR $46 \# 5625$.

DEPARTMENT OF MATHEMATICS, TOKYO METROPOLITAN UNIVERSITY, SETAGAYA-KU, TOKYO, 158, JAPAN 